\title{
Metformin suppresses hepatic gluconeogenesis through induction of SIRT1 and GCN5
}

\author{
Paul W Caton, Nanda K Nayuni, Julius Kieswich, Noorafza Q Khan, Muhammed M Yaqoob \\ and Roger Corder \\ Bart's and the London School of Medicine and Dentistry, William Harvey Research Institute, Queen Mary University, London EC1M 6BQ, UK \\ (Correspondence should be addressed to P W Caton; Email: p.w.caton@qmul.ac.uk)
}

\begin{abstract}
Abnormal elevation of hepatic gluconeogenesis is central to the onset of hyperglycaemia in patients with type 2 diabetes mellitus (T2DM). Metformin corrects hyperglycaemia through inhibition of gluconeogenesis, but its mechanism of action is yet to be fully described. SIRT1 and GCN5 (listed as KAT2A in the MGI Database) have recently been identified as regulators of gluconeogenic gene expression through modulation of levels and activity of the coactivators cAMP-response element binding protein-regulated transcription coactivator 2 (TORC2 or CRTC2 as listed in the MGI Database) and peroxisome proliferator-activated receptor- $\gamma$ coactivator- $1 \alpha$ (PGC1 $\alpha$ or PPARGC1A as listed in the MGI Database). We report that in $\mathrm{db} / \mathrm{db}$ mice, metformin $(250 \mathrm{mg} / \mathrm{kg}$ per day; 7 days) increases hepatic levels of GCN5 protein and mRNA compared with the untreated $\mathrm{db} / \mathrm{db}$ mice, as well as increases levels of SIRT1 protein and activity relative to controls and untreated $\mathrm{db} / \mathrm{db}$ mice. These changes were associated with reduced TORC2 protein level and
\end{abstract}

decreased gene expression and activation of the PGC1 $\alpha$ gene target phosphoenolpyruvate carboxykinase, and lower plasma glucose and insulin. Inhibition of SIRT1 partially blocked the effects of metformin on gluconeogenesis. SIRT1 was increased through an AMP-activated protein kinase-mediated increase in gene expression of nicotinamide phosphoribosyltransferase, the rate-limiting enzyme of the salvage pathway for $\mathrm{NAD}^{+}$. Moreover, levels of GCN5 were dramatically reduced in $\mathrm{db} / \mathrm{db}$ mice compared with the controls. This indicates that loss of GCN5-mediated inhibition of gluconeogenesis appears to constitute a major mechanism for the onset of abnormally elevated hepatic glucose production in $\mathrm{db} / \mathrm{db}$ mice. In conclusion, induction of GCN5 and SIRT1 potentially represents a critical mechanism of action of metformin. In addition, these data identify induction of hepatic GCN5 as a potential therapeutic strategy for treatment of T2DM.

Journal of Endocrinology (2010) 205, 97-106

\section{Introduction}

Prevalence of type 2 diabetes mellitus (T2DM) has increased dramatically over the past four decades. T2DM is characterised by insulin resistance, hyperinsulinaemia and hyperglycaemia. Increased glucose production through abnormally elevated hepatic gluconeogenesis is central to the manifestation of hyperglycaemia in T2DM (Mitrakou et al. 1992, Perriello et al. 1997).

Gluconeogenesis is tightly controlled through the transcriptional regulation of phosphoenolpyruvate carboxykinase (PEPCK; gene code Pck1), the rate-limiting enzyme of hepatic gluconeogenesis, allowing plasma glucose levels to be maintained within a narrow range. PEPCK is abnormally elevated in T2DM (Veneziale et al. 1983), and overexpression of Pck1 mRNA in rodent models is sufficient to produce a T2DM-like state with hyperglycaemia, insulin resistance and hyperinsulinaemia (Valera et al. 1994, Sun et al. 2002). Furthermore, knockdown of Pck1 corrects hyperglycaemia and insulin resistance in $\mathrm{db} / \mathrm{db}$ mice (Gomez-Valades et al. 2006, 2008).
During fasting, gluconeogenic counter-regulatory hormones such as glucagon induce gluconeogenic gene expression. Glucagon signalling causes dephosphorylation and translocation to the nucleus of the cAMP-response element binding protein (CREB)-regulated transcription coactivator 2 (TORC2 also listed as CRCT2 in the MGI Database). In the nucleus, TORC2 coactivates the transcription factor CREB, leading to phosphorylation of CREB at $\mathrm{Ser}^{133}$ and formation of the TORC2-CREB-CBP complex and subsequent induction of peroxisome proliferator-activated receptor- $\gamma$ coactivator- $1 \alpha$ (PGC1 $\alpha$ or PPARGC1A as listed in the MGI Database) an essential coactivator of Pck1 (Yoon et al. 2001, Koo et al. 2005, Dentin et al. 2007). Once activated, PGC1 $\alpha$ forms a complex with the transcription factors Foxo1 and hepatic nuclear factor $4 \alpha$ (Hnf $4 \alpha$ or $H n f 4 a)$, leading to the induction of gluconeogenic genes including Pck1 (Yoon et al. 2001, Puigserver et al. 2003, Rhee et al. 2003). SIRT1, an $\mathrm{NAD}^{+}$-dependent protein deacetylase, inhibits gluconeogenesis through disruption of TORC2 signalling. During fasting TORC2 is acetylated, which protects it against COP1-mediated ubiquitination and subsequent degradation. 
SIRT1 has been reported to deacetylate TORC2, resulting in the loss of protection from ubiquitination, subsequent degradation and ultimately suppression of TORC2-mediated gluconeogenic gene expression (Liu et al. 2008).

In contrast, other studies have reported that SIRT1 functions to activate gluconeogenesis in response to nutrient signalling. SIRT1 interacts with and deacetylates PGC1 $\alpha$ permitting interaction between PGC1 $\alpha, \mathrm{HNF} 4 \alpha$ and FOXO1 leading to the induction of gluconeogenic gene expression. Interestingly, SIRT1-dependent gluconeogenesis was not regulated by classical gluconeogenic regulatory hormones such as glucagon or insulin, but was instead mediated by changes in the levels of $\mathrm{NAD}^{+}$and pyruvate (Rodgers et al. 2005). GCN5 (or KAT2A as listed in the MGI Database), an acetyl transferase, inhibits this process by acetylating and deactivating PGC1 $\alpha$, leading to the suppression of gluconeogenic gene expression (Lerin et al. 2006). In addition, SIRT1 has been reported to deacetylate and stimulate nuclear translocation of FOXO1, leading to the induction of gluconeogenic gene expression (Frescas et al. 2005).

Metformin, the primary therapy for T2DM, ameliorates hyperglycaemia mainly through inhibition of hepatic gluconeogenesis (Hundal et al. 2000). However, its mechanism of action is yet to be fully characterised. We investigated whether metformin inhibits gluconeogenic gene expression by modulating changes in hepatic SIRT1 and GCN5.

\section{Materials and Methods}

\section{Materials}

Mice were obtained from Charles River (Kent, UK). Metformin and all other materials were obtained from Sigma, unless stated otherwise.

\section{Animal experiments}

Eight-week-old $\mathrm{db} / \mathrm{db}$ and control $(\mathrm{db} / \mathrm{m})$ mice were administered metformin $(250 \mathrm{mg} / \mathrm{kg})$ or an equal volume of water every day for 7 days by oral gavage. On day 7 , mice were fasted for $4 \mathrm{~h}$, and blood was collected and centrifuged to obtain plasma. The mice were killed, and livers were removed and snap frozen for protein and mRNA measurements. Animals were maintained on standard chow on a $12 \mathrm{~h}$ light:12 h darkness cycle. All animal experiments were conducted in accordance with the Home Office regulations on the Operation of Animals (Scientific Procedures) Act 1986, published by HMSO, London, UK.

\section{Cell culture}

HepG2 cells were cultured in DMEM containing $5 \mathrm{mM}$ glucose, non-essential amino acids and 10\% (v/v) FCS. Cells were incubated with either metformin (2 mM) or SRT1720 (100 nM; Cayman Chemical, Ann Arbor, MI, USA) with or without compound C (20 $\mu \mathrm{M}$; Calbiochem, Cambridge, UK) in DMEM containing $25 \mathrm{mM}$ glucose for $18 \mathrm{~h}$. For SIRT1 inhibition experiments, HepG 2 cells were incubated for $6 \mathrm{~h}$ with metformin (2 $\mathrm{mM}$ ) with or without 6-chloro-2,3,4,9-tetrahydro-1H-carbazole-1-carboxamide (6TCC; $10 \mu \mathrm{M}$; Alexis Biochemical, Exeter, UK) in DMEM containing $25 \mathrm{mM}$ glucose.

\section{Glucose measurements}

Glucose was measured using a colourimetric glucose oxidase assay based on the method described previously (Caton et al. 2009).

\section{PEPCK activity}

PEPCK activity was assayed as described previously using a three-sequential reaction process based on the stoichiometric transformation of oxaloacetate into ATP (Caton et al. 2009). PEPCK activity is expressed as relative luminescence units.

\section{Quantitative reverse transcription-PCR}

All gene expression was measured using quantitative reverse transcription-PCR according to the procedure described previously (Douthwaite et al. 2003). Expression was determined by $\Delta \Delta C_{\mathrm{t}}$ normalised against $18 \mathrm{~S}$ control RNA.

\section{Immunoblotting}

Solubilised protein samples $(10 \mu \mathrm{g}$; measured and equalised in each fraction using an RC-DC Bio-Rad System (Bio-Rad)) were separated by SDS-PAGE and transferred onto nitrocellulose Hybond membrane or PVDF membrane (GE Healthcare, Amersham, UK). Blots were blocked with 5\% (w/v) milk protein or BSA/Tris-buffered saline plus $0.1 \%$ Tween-20 buffer solution, and were then incubated overnight in primary antibody. Antibodies used in this study are anti-SIRT1 (SCBT, Santa Cruz, CA, USA), anti-GCN5 (Biolegend, San Diego, CA, USA), anti-TORC2 (Calbiochem), anti-phospho $\left(\mathrm{Thr}^{172}\right.$ )-AMP-activated protein kinase (AMPK; Millipore, Billerica, MA, USA), anti-total AMPK, anti-phospho(Ser $\left.{ }^{79}\right)$ ACC, anti-total ACC (all obtained from Cell Signaling Technology, Danvers, MA, USA), anti-FOXO1 (Abcam, Cambridge, UK) and anti-phospho(Ser ${ }^{133}$-CREB (Millipore). Detection of bands was achieved by using the chemiluminescence substrate SuperSignal West Pico (Pierce, Rockford, IL, USA). Reference protein measurements were made with mouse monoclonal anti- $\beta$-actin (clone AC-15) primary antibody in a $3 \%(\mathrm{w} / \mathrm{v})$ milk/TNT solution, at $4{ }^{\circ} \mathrm{C}$.

\section{SIRT1 activity}

SIRT1 activity was assayed in nuclear fractions isolated from mouse liver and HepG2 cells using a two-step fluorometric technique based on deacetylation of the substrate BocLys(Ac)-7-amino-4-methylcoumarin (Boc-Lys(Ac)-AMC; Bachem, St Helens, UK), followed by trypsin treatment AMC (Ishdorj et al. 2008). Nuclear protein isolation was carried out following published methods (Kain et al. 2000). 
Protein content was measured and equalised in each fraction using an RC-DC Bio-Rad System (Bio-Rad). Trichostatin A as a class I and class II histone deacetylase (HDAC) inhibitor was used to confer specificity for SIRT1 activity as opposed to general HDAC activity. SIRT1 activity is expressed as relative fluorescence units.

\section{$N A D^{+} / \mathrm{NADH}$ ratio and ATP levels}

ATP levels and $\mathrm{NAD}^{+} / \mathrm{NADH}$ ratios were measured according to published methods (San et al. 2002, Moynihan et al. 2005).

\section{Statistical analysis}

Results are expressed as mean \pm S.E.M. Statistical comparisons were obtained using StatView (SAS Institute, Inc., Cary, NC, USA). Statistical differences were calculated using either an unpaired $t$-test or one-way ANOVA followed by a Fisher's post-test where appropriate.

\section{Results}

\section{Metformin increases SIRT1 in $\mathrm{db} / \mathrm{db}$ mice}

Systemic activation of SIRT1 with the activator SRT1720 is reported to lower blood glucose and improve insulin sensitivity in Zucker rats and diet-induced obese mice in part through inhibition of hepatic gluconeogenesis (Milne et al. 2007). Therefore, we investigated whether metformin inhibited gluconeogenesis through changes in hepatic SIRT1. Eightweek-old $\mathrm{db} / \mathrm{db}$ or control $(\mathrm{db} / \mathrm{m})$ mice were administered metformin (250 mg/kg per day; 7 days). Levels of SIRT1 protein, activity and $\mathrm{NAD}^{+} / \mathrm{NADH}$ ratio were significantly increased in metformin-treated $\mathrm{db} / \mathrm{db}$ mice compared with the controls and untreated $\mathrm{db} / \mathrm{db}$ mice (Fig. 1A, C and D). Despite increased protein levels, Sirt1 mRNA levels were unchanged following metformin treatment (Fig. 1B). Levels of SIRT1 protein and activity as well as $\mathrm{NAD}^{+} / \mathrm{NADH}$ levels were unchanged between the control and untreated mice (Fig. 1A-C). Metformin had no effect on SIRT1 in control mice (data not shown). Furthermore, incubation of Hep G2 cells with metformin ( $2 \mathrm{mM})$ also resulted in increased levels of SIRT1 protein and activity and $\mathrm{NAD}^{+} / \mathrm{NADH}$ ratio (Fig. 1E-G). This indicates that increasing SIRT1 protein and activity could be a key mechanism by which metformin inhibits gluconeogenic gene expression.

Metformin-induced increases in SIRT1 are associated with lower plasma glucose and insulin through inhibition of gluconeogenesis

We next investigated whether metformin-induced increases in SIRT1 were associated with inhibition of hepatic gluconeogenesis and reduced plasma glucose and insulin. Plasma glucose (Fig. 2A) and insulin (Fig. 2B) levels were significantly elevated in $\mathrm{db} / \mathrm{db}$ mice compared with the
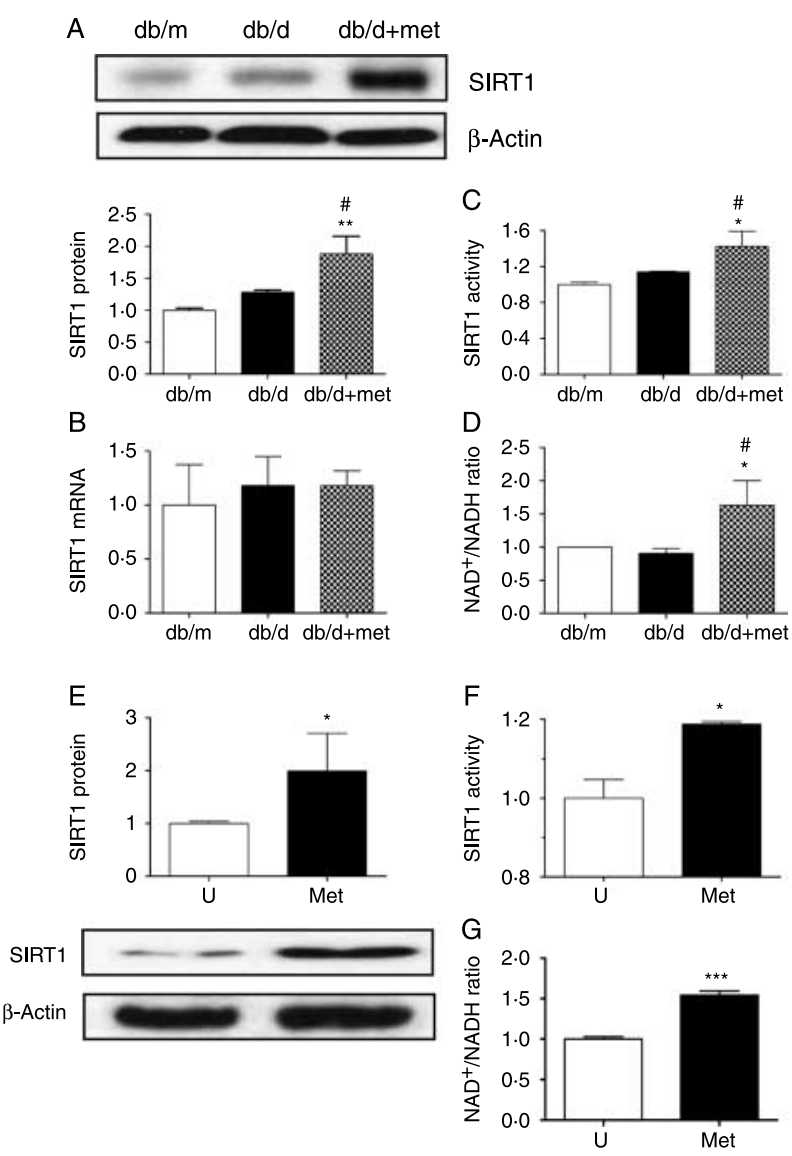

Figure 1 Eight-week-old $\mathrm{db} / \mathrm{d}$ or control $(\mathrm{db} / \mathrm{m})$ mice $(4-8$ per group) were administered metformin ( $\mathrm{db} / \mathrm{d}+$ met; $250 \mathrm{mg} / \mathrm{kg}$; 1 week); (A) SIRT1 protein, (B) SIRT1 activity, (C) NAD ${ }^{+} / \mathrm{NADH}$ ratio, and (D) Sirt1 mRNA. HepG2 cells were incubated with metformin (Met; $2 \mathrm{mM}$ ) for $18 \mathrm{~h}$ in DMEM containing $25 \mathrm{mM}$ glucose; (E) SIRT1 protein, (F) SIRT1 activity and (G) $\mathrm{NAD}^{+} / \mathrm{NADH}$ ratio. Western blots are representative; protein histograms represent pooled densitometry measurements from three to four separate blots. Data are expressed as mean \pm s.E.M. ${ }^{*} P<0 \cdot 05,{ }^{* *} P<0 \cdot 01$, ${ }^{* * *} P<0 \cdot 001$ versus control $(\mathrm{db} / \mathrm{m}$; untreated) or $U$ (untreated HepG2 cells); ${ }^{\sharp} P<0.05$ versus $\mathrm{db} / \mathrm{d}$ (untreated).

controls. These changes were accompanied by significant increases in Pck1 mRNA levels (Fig. 2C) and PEPCK activity (Fig. 2D). Administration of metformin lowered plasma insulin and glucose levels and reduced levels of Pck1 mRNA and PEPCK activity. SIRT1 is reported to inhibit gluconeogenic gene expression through deacetylation and subsequent degradation of TORC2 (Liu et al. 2008). We investigated whether metformin-induced increases in SIRT1 and inhibition of gluconeogenesis were associated with a reduction in TORC2 and $\mathrm{p}\left(\mathrm{Ser}^{133}\right)$-CREB. Levels of TORC2 and $\mathrm{p}\left(\operatorname{Ser}^{133}\right)$-CREB (Fig. $2 \mathrm{E}$ and $\mathrm{F}$ ) protein were increased in $\mathrm{db} / \mathrm{db}$ mice compared with the controls. Consistent with the inhibitory role of SIRT1 on gluconeogenic gene expression, administration of metformin lowered levels of TORC2 and p $\left(\operatorname{Ser}^{133}\right)$-CREB protein (Liu et al. 2008). 

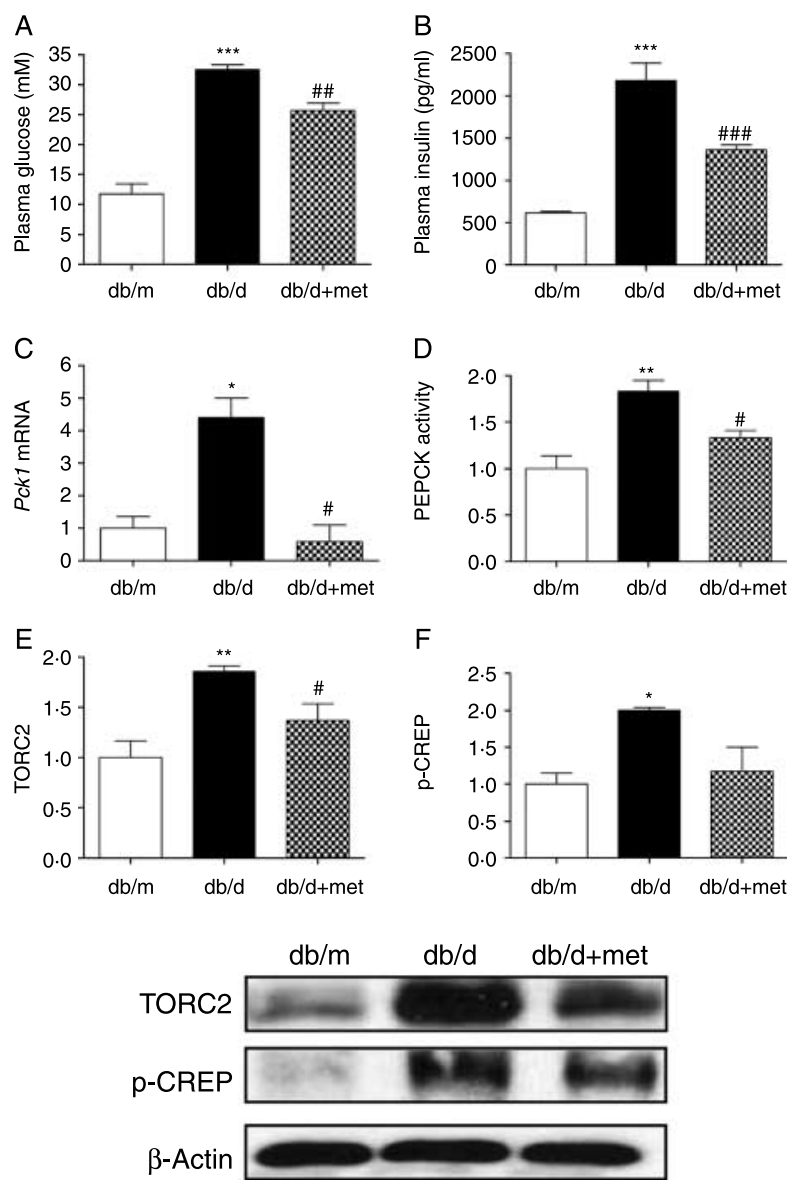

Figure 2 Eight-week-old $\mathrm{db} / \mathrm{d}$ or control $(\mathrm{db} / \mathrm{m})$ mice $(4-8$ per group) were administered metformin (db/d + met; $250 \mathrm{mg} / \mathrm{kg}$; 1 week); (A) Plasma glucose, (B) plasma insulin, (C) Pck1 mRNA, (D) PEPCK activity, (E) TORC2 protein and (F) phospho( $\left.\mathrm{Ser}^{133}\right)$-CREB protein. Western blots are representative; protein histograms represent pooled densitometry measurements from three to four separate blots. Data are expressed as mean \pm S.E.M. $* P<0 \cdot 05$, ${ }^{* *} P<0 \cdot 01,{ }^{* * *} P<0.001$ versus control $(\mathrm{db} / \mathrm{m}$; untreated $) ;{ }^{\#} P<0 \cdot 05$, ${ }^{\#} P<0 \cdot 01,{ }^{\# \#} P<0 \cdot 001$ versus $\mathrm{db} / \mathrm{d}$ (untreated).

\section{Inhibition of SIRT1 blocks metformin-induced inhibition of gluconeogenesis}

To further establish the role of SIRT1 in the gluconeogenic inhibitory action of metformin, HepG2 cells were incubated with metformin and 6TCC, a SIRT1 inhibitor (Napper et al. 2005). 6TCC significantly inhibited SIRT1 activity (Fig. 3A). Metformin (2 $\mathrm{mM}$ ) reduced levels of TORC2 (Fig. 3B) protein in HepG2 cells incubated in DMEM containing $25 \mathrm{mM}$ glucose. Consistent with the role of TORC2 as a key coactivator of gluconeogenic gene expression, metformin also reduced Pck1 gene expression (Fig. 3C), PEPCK activity (Fig. 3D) and glucose levels (Fig. 3E). The effect of metformin on TORC2 protein was completely blocked by 6TCC, while metformin-induced reductions in mRNA levels of Pck1, PEPCK activity and glucose levels were partially blocked by 6TCC.
Metformin increases SIRT1 activity through activation of AMPK

Metformin has been reported to inhibit hepatic gluconeogenesis in part through activation of AMPK (Zhou et al. 2001). Metformin inhibits complex I of the electron transport chain, lowering ATP/AMP ratio (Owen et al. 2000).
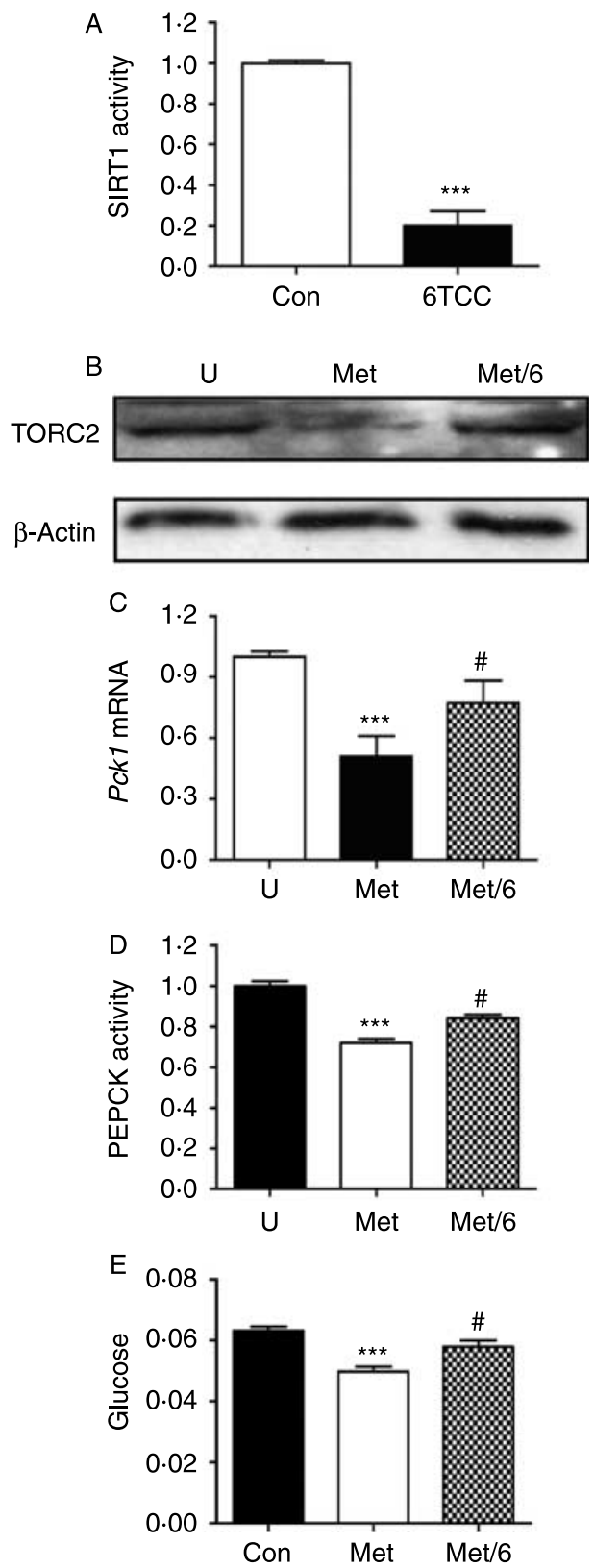

Figure 3 HepG2 cells were incubated with metformin (Met; $2 \mathrm{mM}$ ) with or without 6 TCC $(10 \mu \mathrm{M})$ for $6 \mathrm{~h}$ in DMEM containing $25 \mathrm{mM}$ glucose; (A) SIRT1 activity, (B) TORC2 protein, (C) Pck1 mRNA, (D) PEPCK activity and (E) glucose levels. Western blots are representative. Data are expressed as mean \pm S.E.M. ${ }^{*} P<0 \cdot 05$, ${ }^{* * *} P<0 \cdot 001$ versus $U$ (untreated HepG2); ${ }^{\sharp} P<0 \cdot 05$ versus Met (metformin treated; $2 \mathrm{mM}$ ) HepG2 cells. 
AMP subsequently binds to and allosterically activates AMPK. Moreover, AMP binding induces a conformational change in AMPK, a permissive step for it to act as a substrate for LKB1, which phosphorylates $\mathrm{Thr}^{172}$ on the $\alpha$-subunit of AMPK causing further activation (Hardie 2008). Recent studies have reported that AMPK can increase SIRT1 activity in skeletal myoblasts and skeletal muscle (Fulco et al. 2008, Canto et al. 2009) potentially through an AMPK-mediated increase in the transcription of nicotinamide phosphoribosyltransferase (NAMPT), the rate-limiting enzyme of the salvage pathway for $\mathrm{NAD}^{+}$, an essential co-factor for SIRT1 activity (Landry et al. 2000, Fulco et al. 2008). We investigated whether activation of AMPK by metformin was responsible for increases in SIRT1 in metformin-treated $\mathrm{db} / \mathrm{db}$ mice. Administration of metformin led to decreased hepatic levels of ATP (Fig. 4A) and subsequent increased levels of $\mathrm{p}\left(\mathrm{Thr}^{172}\right)$-AMPK and its downstream target $\mathrm{p}\left(\mathrm{Ser}^{79}\right)$-ACC compared with the control
A

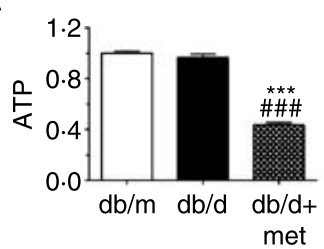

B

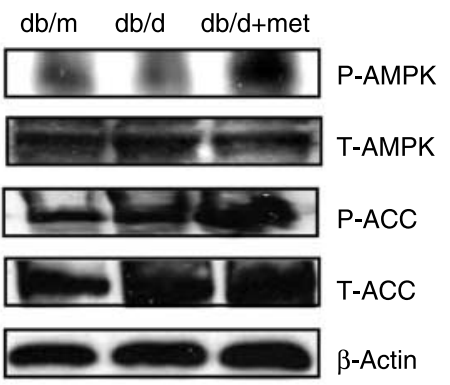

C

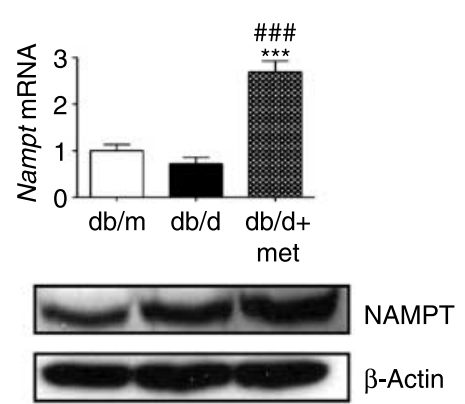

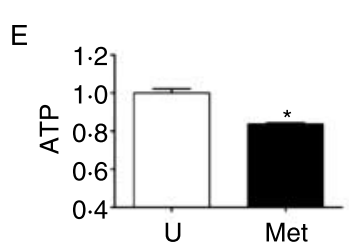

F

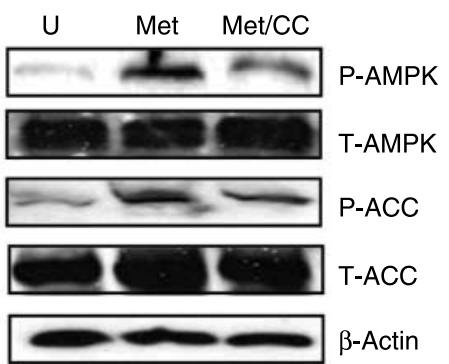

G

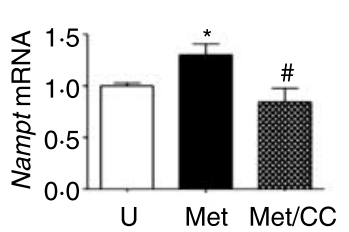

$\mathrm{H}$

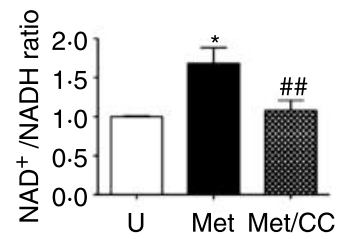

I

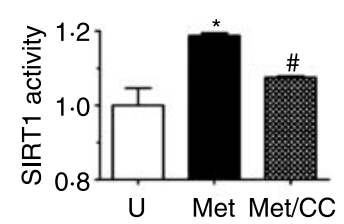

$J$

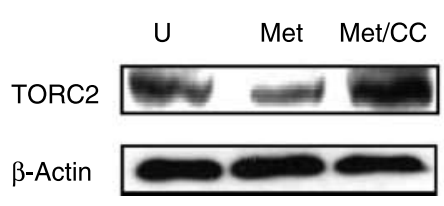

K

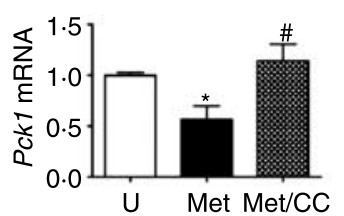

L

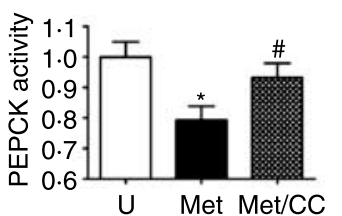

M

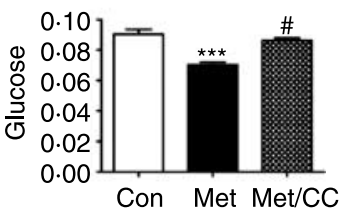

Figure 4 Eight-week-old db/d or control $(\mathrm{db} / \mathrm{m})$ mice (4-8 per group) were administered metformin (db/d+met; $250 \mathrm{mg} / \mathrm{kg}$; $1 \mathrm{week}$ ); (A) Hepatic ATP level, (B) protein levels of phospho(Thr ${ }^{172}$ )-AMPK protein, total AMPK, phospho(Thr $\left.{ }^{172}\right)$-AMPK, $\left(\right.$ Ser $\left.^{79}\right)$-ACC, and total ACC, (C) Nampt mRNA and (D) NAMPT protein. HepG2 cells were incubated with metformin (Met; $2 \mathrm{mM}$ ) with or without compound C (Met/CC; $20 \mu \mathrm{M}$ ) for $18 \mathrm{~h}$ in DMEM containing $25 \mathrm{mM}$ glucose; (E) ATP levels, (F) protein levels of phospho(Thr ${ }^{172}$ )-AMPK protein, total AMPK, phospho(Thr $\left.{ }^{172}\right)$-AMPK, p $\left(S^{79}{ }^{79}\right)$-ACC, total-ACC, (G) Nampt mRNA levels, (H) NAD ${ }^{+} / N_{A D H}$ ratio, (I) SIRT1 activity, (J) TORC2 protein, (K) PCk1 mRNA level, (L) PEPCK activity and (M) glucose level. Western blots are representative. Data are expressed as mean \pm s.E.M. ${ }^{*} P<0 \cdot 05,{ }^{* * *} P<0 \cdot 001$ versus control $\left(\mathrm{db} / \mathrm{m}\right.$; untreated) or $U$ (untreated HepG2); ${ }^{\sharp} P<0 \cdot 05,{ }^{\#} P<0 \cdot 01 ;{ }^{\# \#} P<0 \cdot 001 \mathrm{versus} \mathrm{db} / \mathrm{d}($ untreated) or $\mathrm{M}$ (metformin treated; $2 \mathrm{mM}$ ) HepG2 cells. 
and untreated $\mathrm{db} / \mathrm{db}$ mice (Fig. $4 \mathrm{~B}$ ), indicating activation of AMPK by metformin occurring as a consequence of reduced ATP levels. Levels of total AMPK and ACC were unchanged. In agreement with studies in skeletal myoblasts (Fulco et al. 2008), activation of AMPK was associated with increased levels of Nampt mRNA and NAMPT protein (Fig. 4C and D). Likewise, incubation of HepG2 cells with metformin led to decreased ATP levels (Fig. 4E), increased levels of $\mathrm{p}\left(\mathrm{Thr}^{172}\right)$-AMPK and $\mathrm{p}\left(\mathrm{Ser}^{79}\right)$-ACC (Fig. 4F) and subsequent increased Nampt mRNA levels (Fig. 4G). Increased AMPK activity was associated with increased $\mathrm{NAD}^{+}$, $\mathrm{NADH}$ ratios (Fig. 4H) and SIRT1 activity (Fig. 4I). Compound C, an inhibitor of AMPK, blocked metformininduced increase in protein levels of $\mathrm{p}\left(\mathrm{Thr}^{172}\right)$-AMPK, $\mathrm{p}\left(\mathrm{Ser}^{79}\right)$-ACC, Nampt mRNA, NAD ${ }^{+} / \mathrm{NADH}$ ratio and SIRT1 activity. Consistent with the ability of SIRT1 to facilitate TORC2 degradation, increased $\mathrm{NAD}^{+} / \mathrm{NADH}$ and SIRT1 activity following metformin incubation coincided with reduced protein levels of TORC2 in HepG2 cells (Fig. 4J), as well as with reduced levels of Pck1 mRNA (Fig. 4K), PEPCK activity (Fig. 4L) and glucose levels (Fig. 4M). Incubation with compound $\mathrm{C}$ reversed changes in TORC2 protein, Pck1 mRNA, PEPCK activity and glucose production to basal level. In contrast to changes in SIRT1 activity, metformin-induced increases in SIRT1 protein levels were not inhibited by compound $\mathrm{C}$, indicative of an AMPK-independent mechanism for metformininduced increases in SIRT1 protein levels (Supplementary Figure 1A, see section on supplementary data given at the end of this article). Interestingly, the SIRT1 activator, SRT1720, did not have any effect on the levels of ATP or $\mathrm{p}\left(\mathrm{Thr}^{172}\right)$ AMPK in HepG2 cells (Supplementary Figure 1D and E). In addition, SRT1720 did not increase SIRT1 protein levels nor was the ability to activate SIRT1 inhibited by compound C, indicating that unlike metformin, SRT1720 activates SIRT1 independently of AMPK (Supplementary Figure 1F and G). Incubation of HepG2 cells with compound $\mathrm{C}(20 \mu \mathrm{M})$ alone had no effect on protein levels of total AMPK, total ACC and TORC2, SIRT1 activity, Pck1 mRNA, PEPCK activity and glucose levels. Incubation with compound $\mathrm{C}$ alone led to reduced protein levels of $\mathrm{p}\left(\mathrm{Thr}^{172}\right)$-AMPK and $\mathrm{p}\left(\mathrm{Ser}^{79}\right)$ ACC, consistent with an AMPK-inhibitory action of compound C (Supplementary Figure 2, see section on supplementary data given at the end of this article). Taken together, these data suggest a mechanism of action, whereby metformin potentially increases SIRT1 activity through an AMPK-dependent pathway involving increased Nampt gene expression, leading to inhibition of TORC2-mediated gluconeogenesis. In contrast, metformin-induced increases in SIRT1 protein levels appear to be independent of AMPK.

\section{Metformin induces GNC5 in db/db mice}

Contrary to inhibition of gluconeogenic gene expression through deacetylation of TORC2, SIRT1 has also been reported to induce gluconeogenesis through deacetylation
A $d b / m \quad d b / d \quad d b / d+m e t$

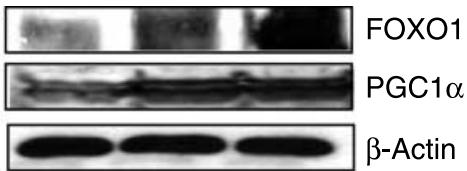

B
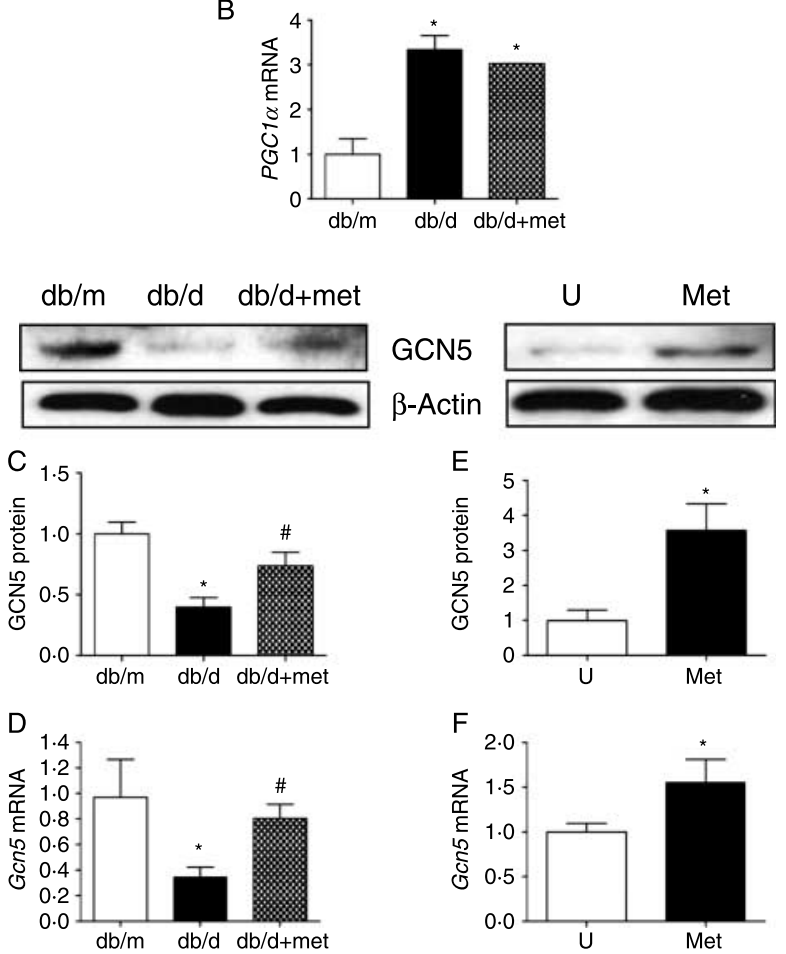

Figure 5 Eight-week-old $\mathrm{db} / \mathrm{d}$ or control $(\mathrm{db} / \mathrm{m})$ mice $(4-8$ per group) were administered metformin ( $\mathrm{db} / \mathrm{db}+$ met; $250 \mathrm{mg} / \mathrm{kg} ; 1$ week); (A) protein levels of FOXO1 and PGC1 $\alpha$, (B) Pgc1 $\alpha$ mRNA, (C) GCN5 protein and (D) Gcn5 mRNA level. HepG2 cells were incubated with metformin (Met; $2 \mathrm{mM}$ ) for $18 \mathrm{~h}$ in DMEM containing 25 mM glucose; (E) GCN5 protein and (F) Gcn5 mRNA. Western blots are representative; protein histograms represent pooled densitometry measurements from three to four separate blots. Data are expressed as mean \pm s.E.M. ${ }^{*} P<0 \cdot 05,{ }^{* *} P<0 \cdot 01$ versus control ( $\mathrm{db} / \mathrm{m}$; untreated) or $U$ (untreated hepG2 cells); ${ }^{\#} P<0 \cdot 05$ versus $\mathrm{db} / \mathrm{d}$ (untreated).

and activation of PGC1 $\alpha$ (Rodgers et al. 2005) and FOXO1 (Frescas et al. 2005) as well as through disruption of signal transducer and activator of transcription 3 (STAT3)dependent inhibition of gluconeogenesis (Nie et al. 2009). Moreover, hepatic knockdown of SIRT1 is reported to lower gluconeogenesis and correct hyperglycaemia in $\mathrm{db} / \mathrm{db}$ mice (Rodgers \& Puigserver 2007, Erion et al. 2009). Figure 1 shows that SIRT1 protein and activity levels and $\mathrm{NAD}^{+}$/ $\mathrm{NADH}$ ratios were unchanged between control and $\mathrm{db} / \mathrm{db}$ mice, indicating that increased hepatic gluconeogenesis in $\mathrm{db} / \mathrm{db}$ mice was not caused by increased SIRT1. However, metformin-induced increases in SIRT1 could potentially lead to induction of gluconeogenesis in metformin-administered 


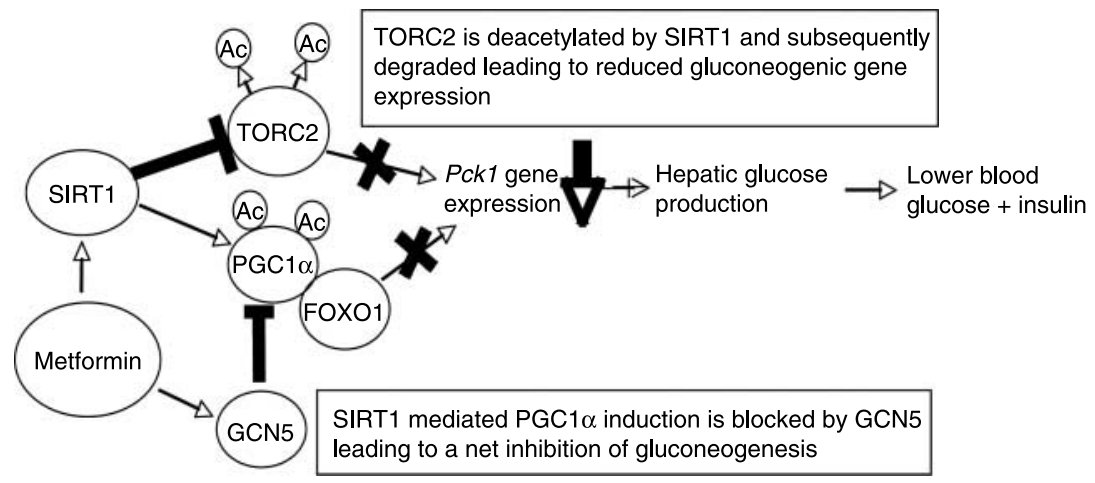

Figure 6 Schematic representation of the proposed mechanism of inhibition of gluconeogenic gene expression by metformin, GCN5 and SIRT1.

$\mathrm{db} / \mathrm{db}$ mice. Given the ability of SIRT1 to induce PGC1 $\alpha$ and FOXO1-dependent Pck1 gene expression, we measured the impact of metformin administration on Pgc1 $1 \alpha$ mRNA and PGC1 $\alpha$ protein and FOXO1 protein in $\mathrm{db} / \mathrm{db}$ mice. Levels of FOXO1 protein and PGC1 $\alpha$ (Fig. 5A) and $P g c 1 \alpha$ mRNA (Fig. 5B) protein were increased in $\mathrm{db} / \mathrm{db}$ mice compared with the $\mathrm{db} / \mathrm{m}$ controls. However, despite lower plasma glucose levels and reduced protein levels of TORC2 and $\mathrm{p}\left(\mathrm{Ser}^{133}\right)$-CREB (Fig. 2), levels of Pgc1 $\alpha$ mRNA, PGC1 $\alpha$ protein and FOXO1 protein were not reduced following metformin administration, consistent with the putative stimulatory effects of SIRT1 on gluconeogenic gene expression.

SIRT1-dependent deacetylation and activation of PGC1 $\alpha$ and subsequent induction of Pck1 gene expression are blocked by GCN5. Also, overexpression of GCN5 decreases gluconeogenic gene expression and lowers plasma glucose levels in fasted mice, without changes in $P g c 1 \alpha$ mRNA levels (Lerin et al. 2006). However, the contribution of altered GCN5 levels to the onset of insulin resistance and T2DM is yet to be investigated. GCN5 protein and mRNA levels were significantly decreased in $\mathrm{db} / \mathrm{db}$ mice compared with the controls, but they returned to basal level following metformin administration (Fig. 5C and D), indicating that reduced GCN5 levels and the associated loss of inhibition of gluconeogenic gene expression play a key role in the manifestation of abnormal hepatic glucose production in T2DM. Incubation of HepG2 cells with metformin also led to increased levels of GCN5 protein (Fig. 5E) and mRNA (Fig. 5F). However, metformin-induced increases in levels of GCN5 protein and mRNA were not reversed by compound C (Supplementary Figure 1B and C). Taken together, these data suggest that metformin can induce expression of GCN5 protein and mRNA, and that following metformin administration, potential SIRT1-dependent increases in gluconeogenic gene expression are blocked by these increases in GCN5 (Fig. 6), leading to a net decrease in gluconeogenesis and hepatic glucose output.

\section{Discussion}

Metformin, the primary therapeutic agent for T2DM patients, corrects hyperglycaemia and hyperinsulinaemia predominantly through its ability to lower hepatic gluconeogenesis (Hundal et al. 2000). Despite being in clinical use for over 50 years (He et al. 2009, Wong et al. 2009), its exact mechanism of action is yet to be fully characterised. Here, we report that metformin increases hepatic GCN5 and SIRT1, and that the ability to induce these key regulators of gluconeogenic gene expression potentially represents an important mechanism of the action of metformin. We propose a model whereby metformin increases SIRT1 protein and activity, leading to the suppression of TORC2-mediated gluconeogenesis, while the potential stimulatory effects of SIRT1 on gluconeogenesis are countered by metformindependent induction of GCN5, which would oppose SIRT1-dependent PGC1 $\alpha$ activation (Lerin et al. 2006). This results in a net inhibition of gluconeogenic gene expression and reduced hepatic glucose production. Interestingly, SIRT1-mediated induction of gluconeogenesis was reported to be activated in response to nutrient signalling, but it was unaffected by glucagon, suggesting that there exist parallel hormonal and nutrient pathways for induction of gluconeogenesis (Rodgers et al. 2005, 2008). Thus, induction of both GCN5 and SIRT1 could represent a mechanism by which metformin can inhibit both hormone- and nutrientinduced gluconeogenic gene expression.

Recent studies have highlighted links between SIRT1 and another conserved regulator of metabolism, AMPK. We report that activation of hepatic AMPK by metformin induces increased expression of NAMPT mRNA and protein, and consequent increased $\mathrm{NAD}^{+} / \mathrm{NADH}$ ratio and SIRT1 activity, leading to the inhibition of TORC2-mediated gluconeogenesis. Hence, despite the potential reduction in $\mathrm{NAD}^{+} / \mathrm{NADH}$ ratio that could stem from metformin inhibition of complex I (Owen et al. 2000), consequent AMPK-mediated increases in NAMPT appear to result in a net increase in $\mathrm{NAD}^{+} / \mathrm{NADH}$ ratio and activation of 
SIRT1. This process is similar to that previously described in skeletal myoblasts, where AMPK activates SIRT1 via the induction of NAMPT (Fulco et al. 2008). However, different convergent roles of SIRT1 and AMPK have also been identified in the liver. Recent studies have reported that SIRT1 can deacetylate and activate LKB1, leading to the activation of AMPK (Hou et al. 2008, Lan et al. 2008). This finding and the results presented here raise the possibility that a positive feedback system could be operative in response to hyperglycaemia following metformin administration, where activation of AMPK leads to the activation of SIRT1. In turn, SIRT1 deacetylates LKB1, leading to further activation of AMPK. However, further study is required to fully elucidate the relationship between hepatic SIRT1 and AMPK, and their roles in the regulation of hepatic glucose metabolism.

AMPK-mediated TORC2 phosphorylation $\left(\mathrm{Ser}^{171}\right)$ and nuclear exclusion have been suggested as mechanisms of action of metformin (Shaw et al. 2005). However, since TORC2 is O-glycosylated at $\operatorname{Ser}^{171}$ in insulin resistance, making phosphorylation impossible and resulting in nuclear retention (Dentin et al. 2008), this is unlikely to represent a true mechanism of action of metformin in T2DM (He et al. 2009). In contrast, the data presented here indicate that AMPK-mediated activation of SIRT1 and consequent TORC2 degradation have greater potential as likely mechanisms of the action of metformin. This likely occurs in parallel with metformin-induced phosphorylation of CBP, which was recently reported to cause dissociation of the CREB-TORC2-CBP complex (He et al. 2009) and AMPKmediated induction of the nuclear receptor SHP, which has been shown to mediate metformin-directed inhibition of gluconeogenic gene expression (Kim et al. 2008).

Interestingly, SRT1720, a SIRT1 activator currently under investigation as a treatment for T2DM (Milne et al. 2007), does not appear to increase SIRT1 activity through an AMPK-mediated mechanism. Further study is required to elucidate the mechanisms by which these compounds correct hyperglycaemia, seemingly without stimulating gluconeogenesis. In addition, this study also provides a potential mechanism of action by which polyphenolic compounds such as resveratrol might activate SIRT1 since they appear to activate both SIRT1 and AMPK (Zang et al. 2006, Hou et al. 2008). Indeed, a recent study has reported that AMPK - / mice are resistant to the metabolic effects of resveratrol, suggesting that the primary target of resveratrol is AMPK and not SIRT1 (Um et al. 2010).

This study has highlighted a possible mechanism of metformin action, involving increased SIRT1 activity through the activation of AMPK. However, we also observed metformin-dependent increases in the levels of SIRT1 protein, and perhaps more importantly, GCN5 protein and mRNA that were seemingly independent of AMPK. The fact that Sirt1 mRNA levels were unchanged while SIRT1 protein levels were significantly increased is indicative of a post-translational mechanism being responsible for increased protein levels. Recent studies have shown that phosphorylation of SIRT1 by c-Jun N-terminal protein kinase 2 (JNK2; Ford et al. 2008), cyclin B/CDK1 and potentially other kinases (Sasaki et al. 2008) can increase stability and extend half-life of SIRT1 protein. SIRT1 protein levels were not regulated by AMPK in this system, and further work is required to identify the kinase system potentially responsible for SIRT1 phosphorylation following metformin administration. Moreover, further investigation is required to elucidate the mechanisms responsible for metformin-induced increase in Gcn 5 mRNA and GCN5 protein levels.

In conclusion, we have identified that increases in GCN5 and SIRT1 potentially represent a mechanism by which metformin inhibits hepatic gluconeogenesis. Moreover, loss of GCN5-mediated inhibition of gluconeogenesis could constitute a major mechanism for the onset of abnormally elevated hepatic glucose production in $\mathrm{db} / \mathrm{db}$ mice. Therefore, strategies aimed at increasing hepatic GCN5 levels may represent potential therapeutic targets for the treatment of T2DM, and further investigation is required to understand GCN5 regulation in the onset of hyperglycaemia in T2DM.

\section{Supplementary data}

This is linked to the online version of the paper at http://dx.doi.org/10.1677/ JOE-09-0345.

\section{Declaration of interest}

The authors declare that there is no conflict of interest that could be perceived as prejudicing the impartiality of the research reported.

\section{Funding}

This research did not receive any specific grant from any funding agency in the public, commercial or not-for-profit sector.

\section{References}

Canto C, Gerhart-Hines Z, Feige JN, Lagouge M, Noriega L, Milne JC, Elliott PJ, Puigserver P \& Auwerx J 2009 AMPK regulates energy expenditure by modulating $\mathrm{NAD}^{+}$metabolism and SIRT1 activity. Nature 458 1056-1060.

Caton PW, Nayuni NK, Murch O \& Corder R 2009 Endotoxin induced hyperlactatemia and hypoglycemia is linked to decreased mitochondrial phosphoenolpyruvate carboxykinase. Life Sciences 84 738-744.

Dentin R, Liu Y, Koo SH, Hedrick S, Vargas T, Heredia J, Yates J III \& Montminy M 2007 Insulin modulates gluconeogenesis by inhibition of the coactivator TORC2. Nature 449 366-369.

Dentin R, Hedrick S, Xie J, Yates J III \& Montminy M 2008 Hepatic glucose sensing via the CREB coactivator CRTC2. Science 319 1402-1405.

Douthwaite JA, Lees DM \& Corder R 2003 A role for increased mRNA stability in the induction of endothelin-1 synthesis by lipopolysaccharide. Biochemical Pharmacology 66 589-594. 
Erion DM, Yonemitsu S, Nie Y, Nagai Y, Gillum MP, Hsiao JJ, Iwasaki T, Stark R, Weismann D, Yu XX et al. 2009 SirT1 knockdown in liver decreases basal hepatic glucose production and increases hepatic insulin responsiveness in diabetic rats. PNAS 106 11288-11293.

Ford J, Ahmed S, Allison S, Jiang M \& Milner J 2008 JNK2-dependent regulation of SIRT1 protein stability. Cell Cycle 7 3091-3097.

Frescas D, Valenti L \& Accili D 2005 Nuclear trapping of the forkhead transcription factor FoxO1 via Sirt-dependent deacetylation promotes expression of glucogenetic genes. Journal of Biological Chemistry $\mathbf{2 8 0}$ 20589-20595.

Fulco M, Cen Y, Zhao P, Hoffman EP, McBurney MW, Sauve AA \& Sartorelli V 2008 Glucose restriction inhibits skeletal myoblast differentiation by activating SIRT1 through AMPK-mediated regulation of Nampt. Developmental Cell 14 661-673.

Gomez-Valades AG, Vidal-Alabro A, Molas M, Boada J, Bermudez J, Bartrons R \& Perales JC 2006 Overcoming diabetes-induced hyperglycemia through inhibition of hepatic phosphoenolpyruvate carboxykinase (GTP) with RNAi. Molecular Therapy 13 401-410.

Gomez-Valades AG, Mendez-Lucas A, Vidal-Alabro A, Blasco FX, Chillon M, Bartrons R, Bermudez J \& Perales JC 2008 Pck1 gene silencing in the liver improves glycemia control, insulin sensitivity, and dyslipidemia in $\mathrm{db} / \mathrm{db}$ mice. Diabetes 57 2199-2210.

Hardie DG 2008 Role of AMP-activated protein kinase in the metabolic syndrome and in heart disease. FEBS Letters 582 81-89.

He L, Sabet A, Djedjos S, Miller R, Sun X, Hussain MA, Radovick S \& Wondisford FE 2009 Metformin and insulin suppress hepatic gluconeogenesis through phosphorylation of CREB binding protein. Cell 137 635-646.

Hou X, Xu S, Maitland-Toolan KA, Sato K, Jiang B, Ido Y, Lan F, Walsh K, Wierzbicki M, Verbeuren TJ et al. 2008 SIRT1 regulates hepatocyte lipid metabolism through activating AMP-activated protein kinase. Journal of Biological Chemistry 283 20015-20026.

Hundal RS, Krssak M, Dufour S, Laurent D, Lebon V, Chandramouli V, Inzucchi SE, Schumann WC, Petersen KF, Landau BR et al. 2000 Mechanism by which metformin reduces glucose production in type 2 diabetes. Diabetes 49 2063-2069.

Ishdorj G, Graham BA, Hu X, Chen J, Johnston JB, Fang X \& Gibson SB 2008 Lysophosphatidic acid protects cancer cells from histone deacetylase (HDAC) inhibitor-induced apoptosis through activation of HDAC Journal of Biological Chemistry 283 16818-16829.

Kain KH, Popov VL \& Herzog NK 2000 Alterations in mitochondria and mtTFA in response to LPS-induced differentiation of B-cells. Biochimica et Biophysica Acta 1494 91-103.

Kim YD, Park KG, Lee YS, Park YY, Kim DK, Nedumaran B, Jang WG, Cho WJ, Ha J, Lee IK et al. 2008 Metformin inhibits hepatic gluconeogenesis through AMP-dependent protein kinase-dependent regulation of orphan nuclear receptor SHP. Diabetes 57 306-314.

Koo SH, Flechner L, Qi L, Zhang X, Screaton RA, Jeffries S, Hedrick S, $\mathrm{Xu}$ W, Boussouar F, Brindle P et al. 2005 The CREB coactivator TORC2 is a key regulator of fasting glucose metabolism. Nature $\mathbf{4 3 7}$ 1109-1111.

Lan F, Cacicedo JM, Ruderman N \& Ido Y 2008 SIRT1 modulation of the acetylation status, cytosolic localization, and activity of LKB1. Possible role in AMP-activated protein kinase activation. Journal of Biological Chemistry $28327628-27635$.

Landry J, Sutton A, Tafrov ST, Heller RC, Stebbins J, Pillus L \& Sternglanz R 2000 The silencing protein SIR2 and its homologs are NAD-dependent protein deacetylases. PNAS 97 5807-5811.

Lerin C, Rodgers JT, Kalume DE, Kim SH, Pandey A \& Puigserver P 2006 GCN5 acetyltransferase complex controls glucose metabolism through transcriptional repression of PGC-1 $\alpha$. Cell Metabolism 3 429-438.

Liu Y, Dentin R, Chen D, Hedrick S, Ravnskjaer K, Schenk S, Milne J, Meyers DJ, Cole P, Yates J III et al. 2008 A fasting inducible switch modulates gluconeogenesis via activator/coactivator exchange. Nature $\mathbf{4 5 6}$ 269-273

Milne JC, Lambert PD, Schenk S, Carney DP, Smith JJ, Gagne DJ, Jin L, Boss O, Perni RB, Vu CB et al. 2007 Small molecule activators of SIRT1 as therapeutics for the treatment of type 2 diabetes. Nature $\mathbf{4 5 0}$ $712-716$
Mitrakou A, Kelley D, Mokan M, Veneman T, Pangburn T, Reilly J \& Gerich J 1992 Role of reduced suppression of glucose production and diminished early insulin release in impaired glucose tolerance. New England Journal of Medicine 326 22-29.

Moynihan KA, Grimm AA, Plueger MM, Bernal-Mizrachi E, Ford E, Cras-Meneur C, Permutt MA \& Imai S 2005 Increased dosage of mammalian Sir2 in pancreatic beta cells enhances glucose-stimulated insulin secretion in mice. Cell Metabolism 2 105-117.

Napper AD, Hixon J, McDonagh T, Keavey K, Pons JF, Barker J, Yau WT, Amouzegh P, Flegg A, Hamelin E et al. 2005 Discovery of indoles as potent and selective inhibitors of the deacetylase SIRT1. Journal of Medicinal Chemistry 48 8045-8054.

Nie Y, Erion DM, Yuan Z, Dietrich M, Shulman GI, Horvath TL \& Gao Q 2009 STAT3 inhibition of gluconeogenesis is downregulated by SirT1. Nature Cell Biology 11 492-500.

Owen MR, Doran E \& Halestrap AP 2000 Evidence that metformin exerts its anti-diabetic effects through inhibition of complex 1 of the mitochondrial respiratory chain. Biochemical Journal 348 607-614.

Perriello G, Pampanelli S, Del Sindaco P, Lalli C, Ciofetta M, Volpi E, Santeusanio F, Brunetti P \& Bolli GB 1997 Evidence of increased systemic glucose production and gluconeogenesis in an early stage of NIDDM. Diabetes 46 1010-1016.

Puigserver P, Rhee J, Donovan J, Walkey CJ, Yoon JC, Oriente F, Kitamura Y, Altomonte J, Dong H, Accili D et al. 2003 Insulin-regulated hepatic gluconeogenesis through FOXO1-PGC-1 $\alpha$ interaction. Nature $\mathbf{4 2 3}$ $550-555$.

Rhee J, Inoue Y, Yoon JC, Puigserver P, Fan M, Gonzalez FJ \& Spiegelman BM 2003 Regulation of hepatic fasting response by PPARgamma coactivator-1 $\alpha$ (PGC-1): requirement for hepatocyte nuclear factor $4 \alpha$ in gluconeogenesis. PNAS 100 4012-4017.

Rodgers JT \& Puigserver P 2007 Fasting-dependent glucose and lipid metabolic response through hepatic sirtuin 1. PNAS 104 12861-12866.

Rodgers JT, Lerin C, Haas W, Gygi SP, Spiegelman BM \& Puigserver P 2005 Nutrient control of glucose homeostasis through a complex of PGC- $1 \alpha$ and SIRT1. Nature 434 113-118.

Rodgers JT, Lerin C, Gerhart-Hines Z \& Puigserver P 2008 Metabolic adaptations through the PGC- $1 \alpha$ and SIRT1 pathways. FEBS Letters $\mathbf{5 8 2}$ $46-53$.

San KY, Bennett GN, Berrios-Rivera SJ, Vadali RV, Yang YT, Horton E, Rudolph FB, Sariyar B \& Blackwood K 2002 Metabolic engineering through cofactor manipulation and its effects on metabolic flux redistribution in Escherichia coli. Metabolic Engineering 4 182-192.

Sasaki T, Maier B, Koclega KD, Chruszcz M, Gluba W, Stukenberg PT, Minor W \& Scrable H 2008 Phosphorylation regulates SIRT1 function. PLoS ONE 3 e4020.

Shaw RJ, Lamia KA, Vasquez D, Koo SH, Bardeesy N, Depinho RA, Montminy M \& Cantley LC 2005 The kinase LKB1 mediates glucose homeostasis in liver and therapeutic effects of metformin. Science $\mathbf{3 1 0}$ 1642-1646.

Sun Y, Liu S, Ferguson S, Wang L, Klepcyk P, Yun JS \& Friedman JE 2002 Phosphoenolpyruvate carboxykinase overexpression selectively attenuates insulin signaling and hepatic insulin sensitivity in transgenic mice. Journal of Biological Chemistry 277 23301-23307.

Um JH, Park SJ, Kang H, Yang S, Foretz M, McBurney MW, Kim MK, Viollet B \& Chung JH 2010 AMPK-deficient mice are resistant to the metabolic effects of resveratrol. Diabetes 59 554-563.

Valera A, Pujol A, Pelegrin M \& Bosch F 1994 Transgenic mice overexpressing phosphoenolpyruvate carboxykinase develop non-insulindependent diabetes mellitus. PNAS 91 9151-9154.

Veneziale CM, Donofrio JC \& Nishimura H 1983 The concentration of P-enolpyruvate carboxykinase protein in murine tissues in diabetes of chemical and genetic origin. Journal of Biological Chemistry 258 14257-14262.

Wong AK, Howie J, Petrie JR \& Lang CC 2009 AMP-activated protein kinase pathway: a potential therapeutic target in cardiometabolic disease. Clinical Science 116 607-620. 
Yoon JC, Puigserver P, Chen G, Donovan J, Wu Z, Rhee J, Adelmant G, Stafford J, Kahn CR, Granner DK et al. 2001 Control of hepatic gluconeogenesis through the transcriptional coactivator PGC-1. Nature $\mathbf{4 1 3}$ 131-138.

Zang M, Xu S, Maitland-Toolan KA, Zuccollo A, Hou X, Jiang B, Wierzbicki M, Verbeuren TJ \& Cohen RA 2006 Polyphenols stimulate AMP-activated protein kinase, lower lipids, and inhibit accelerated atherosclerosis in diabetic LDL receptor-deficient mice. Diabetes $\mathbf{5 5}$ 2180-2191.
Zhou G, Myers R, Li Y, Chen Y, Shen X, Fenyk-Melody J, Wu M, Ventre J, Doebber T, Fujii N et al. 2001 Role of AMP-activated protein kinase in mechanism of metformin action. Journal of Clinical Investigation 108 1167-1174.

Received in final form 19 December 2009

Accepted 20 January 2010

Made available online as an Accepted Preprint 20 January 2010 